\title{
LATTICE POINTS AND LIE GROUPS. III
}

\author{
ROBERT S. CAHN
}

ABSTR ACT. If a compact, simply connected, semisimple Lie group is considered as a Riemannian manifold with metric arising from the negative of the Killing form it is shown that its volume is

$$
(4 \pi)^{\operatorname{dim} G / 2} \Gamma(\operatorname{dim} G / 2+1)(1 /|w|) \int_{|\mathbf{\Lambda}| \leq 1} f^{2}(\mathbf{\Lambda}) d \mathbf{\Lambda} .
$$

1. Introduction. Let $(M, g)$ be a compact Riemannian manifold, $M$, with metric $g$ and Laplacian $\Delta$. If $0=\lambda_{1} \leq \lambda_{2} \leq \cdots$ are the eigenvalues of $-\Delta$ it is well known from the results of Minakshisundaram and Pleijel [3] that $Z(t)=\Sigma_{1}^{\infty} e^{-\lambda_{n} t}$, the zeta-function of $M$, is asymptotic to (Riemannian volume of $M) /(4 \pi t)^{\operatorname{dim} M / 2}$ as $t \downarrow 10$. In this paper we will make specific calculations of the volume for the case of a compact, connected, simply connected, semisimple Lie group $G$. We denote by $e$ the identity element of the group $G$ and by $g$ the Lie algebra of $G$. The metric $g$ will act on the tangent space at $e$, i.e. $g$, as the negative of the Killing form and $g$ will be extended to the entire group by left translation. Before proceeding further the author wishes to thank Burton Randol and Joseph Wolf for their observations.

2. $Z(t)$. Having fixed the metric $g$, we wish to compute $Z(t)$. If we view the elements of the universal enveloping algebra of $g$ as left-invariant differential operators, the negative of the Casimir operator, $C$, becomes the Laplacian $\Delta$ of $(G, g)$. We now mention a few properties of $C$. For any representation $\pi$ of $G$ (or equivalently $g$ ) $\pi(C)$ acts diagonally. Using the bijection between the irreducible representations of $G$ and dominant integral weights, $\lambda$, in the dual of a Cartan subalgebra, $\bar{b}$, of $g_{C}=g \otimes \mathbf{C}$, we will write $\pi_{\lambda}$ for the representation with dominant weight $\lambda$. Then

Received by the editors July 27, 1973 and, in revised form, September 12, 1973 and October 12, 1973.

AMS(MOS) subject classifications (1970). Primary 35P20, $58 \mathrm{G} 05$.

Key words and phrases. Compact semisimple group, Casimir operator, Laplacian, zeta function. 
$\pi_{\lambda}(C)=\left(|\Lambda|^{2}-|\delta|^{2}\right)$ Id where $\Lambda=\lambda+\delta, \delta=1 / 2$ the sum of the positive roots of $g_{C}$ and norms are taken with respect to the Killing form. Now using the Peter-Weyl theorem and the Weyl character formula as in [1]

$$
Z(t)=\sum_{\Lambda>0} f^{2}(\Lambda) \exp \left\{-\left(|\Lambda|^{2}-|\delta|^{2}\right) t\right\}=\frac{1}{|w|} \sum_{\Lambda} f^{2}(\Lambda) \exp \left\{-\left(|\Lambda|^{2}-|\delta|^{2}\right) t\right\}
$$

where $f(\Lambda)=\Pi_{a>0}(\Lambda, a) / \Pi_{a>0}(\delta, \alpha)$ and $|w|$ is the order of the Weyl group.

3. Calculation of the volume. To analyze $Z(t)$ we use an Abelian theorem. We define a measure $d m(\lambda)$ on $(0, \infty)$ as a sum of Dirac delta functions

$$
d m(\lambda)=\sum_{y=|\mathbf{\Lambda}|^{2}-|\delta|^{2}} c_{y} \delta_{y}(\lambda) \text { where } c_{y}=\frac{1}{|w|} \sum_{\mathbf{\Lambda}:|\mathbf{\Lambda}|^{2}-|\delta|^{2}=y} f^{2}(\Lambda) .
$$

Then $Z(t)=\int_{0}^{\infty} e^{-\lambda} d m(\lambda)$, the Laplace transform of $d m(\lambda)$. If $m(x)=$ $\int_{0}^{x} d m(\lambda)$, then from [1]

$$
m(x) \sim x^{\operatorname{dim} G / 2} \frac{1}{|w|} \int_{|\Lambda| \leq 1} f^{2}(\Lambda) d \Lambda,
$$

where $d \Lambda$ is Haar measure on the real span of the roots in the dual of 5 normalized such that the volume of the parallelepiped spanned by the fundamental weights equals 1 .

Using an Abelian theorem [2, p. 420]

$$
\begin{aligned}
Z(t) & \sim \Gamma(\operatorname{dim} G / 2+1) m(1 / t) \quad(t \downarrow 0) \\
& \sim \Gamma(\operatorname{dim} G / 2+1) \frac{1}{t^{\operatorname{dim} G / 2}} \frac{1}{|w|} \int_{|\Lambda| \leq 1} f^{2}(\Lambda) d \Lambda .
\end{aligned}
$$

Using the results of [3]

$$
Z(t) \sim(\text { Volume } G) /(4 \pi t)^{\operatorname{dim} G / 2} .
$$

Thus

Volume $G=(4 \pi)^{\operatorname{dim} G / 2} \Gamma(\operatorname{dim} G / 2+1) \frac{1}{|w|} \int_{|\Lambda| \leq 1} f^{2}(\Lambda) d \Lambda$. 


\section{BIBLIOGRAPHY}

1. R. S. Cahn, Lattice points and Lie groups. II, Trans. Amer. Math. Soc. 183 (1973), 131-137.

2. W. Feller, An introduction to probability and its applications. Vol. II, Wiley, New York, 1966. MR 35 \#1048.

3. S. Minak shisundaram and A. Pleijel, Some properties of the eigenfunctions of the Laplace-operator on Riemannian manifolds, Canad. J. Math. 1 (1949), 242256. MR 11, 108.

DEPARTMENT OF MATHEMATICS, UNIVERSITY OF MIAMI, CORAL GABLES, FLORIDA 33124 